\title{
Ein Fall von absichtlich vorgetäuschter kataleptischer Totenstarre ${ }^{1}$ ).
}

Von

Prof. Dr. med. Th. Lochte, Göttingen.

Yit 1 Textabbildung.

Unter kataleptischer Totenstarre versteht man bekanntlich die Fortdauer einer während des Lebens eingetretenen Kontraktion der Muskeln über den Tod hinaus bis zum Eintritt der Totenstarre. Sie wurde beobachtet bei schweren Verletzungen des Zentralnervensystems, insbesondere nach Schußverletzungen in der hinteren Schädelgrube. Dementsprechend hat man sie oft im Kriege beobachtet, zuerst im Krimkriege; später sind derartige Beobachtungen unter anderen von Rossbach aus dem Kriege 1870-1871 mitgeteilt worden.

In anderen Fällen hat man sie nach Krampfzuständen beobachtet. Schlesinger sah bei 2 mit Magendilatation und Tetanie behafteter Personen den tetanischen Zustand der Arme den Tod überdauern; man hat auch bei Tetanus, ferner bei Erstickung, z. B. bei Ertrunkenen und bei der Kohlenoxydvergiftung die kataleptische Totenstarre beobachtet; schließlich ist sie bei plötzlichen Todesfällen, z. B. bei Schuß in die Lungen oder in das Herz gesehen worden, insbesondere bei Blitzverletzungen.

Dagegen kommen auch ungewöhnliche Haltungen des Körpers vor, die zufällig im Momente des Todes fixiert sind. Wenn z. B. ein im Anschlag liegender Soldat durch einen Schädelschuß schnell getötet wird, and nun in der Anschlagstellung erstart, so liegt deshalb noch keine kataleptische Totenstarre vor. Der Körper befand sich eben nur zufällig in dieser Lage und mußte in derselben erstarren. Wir sprechen clann von einer zufällig vorgetäuschten kataleptischen Totenstarre.

Von einer absichtlich vorgetäuschten kataleptischen Totenstarre ist in der Literatur nichts bekannt. Nur gelegentlich hat man wohl angenommen, daß die Schußwaffe der Leiche nachträglich in die Hand gegeben sei (vgl. Lacassagne, Archives d'anthropolog. crim. 9, 135; 1894).

Der nachfolgende Fall verdient nach mehreren Richtungen hin Interesse :

1) Vorgetragen auf der Tagung der Deutschen Gesellschaft für gerichtliche und soziale Medizin in Erlangen, September 1921. 
In einer großen Provinzialstadt war in einer Etagenwohnung bei Herrn Z. am 2.November eine ca. 35 jährige Frau N. mit ihren beiden 7- und 10 jährigen Töchtern einquartiert worden. Man hatte den Mietern zwei Zimmer ïberwiesen. Das einfenstrige Schlafzimmer hatte einen nicht verschließbaren Eingang vom Flur aus. Frau N. war gern gesehen im Hause. Sie half bei den verschiedensten Arbeiten. Sie speiste mit ihren Kindern gemeinsam mit den Wirtsleuten; bestehend aus dem Hausherrn, dessen Ehefrau, einer erwachsenen Tochter und einem Gymnasiasten (Primaner). Der Ehemann der Frau N. war nach Brasilien gereist, um dort event. eine Farm zu kaufen. Im Haushalt befand sich noch das Dienstmädchen K. Anfang Januar 1921 war die Hausfrau mit ihrer Tochter verreist.

Am 6. Januar abends besuchte Herr Z. ein Theater, das Dienstmädchen war ausgegangen, Frau $\mathrm{N}$. mit ihren beiden Töchtern und der Sehüler befanden sich allein in der Wohnung.

Die Zeugen geben an, daß in der Wohnung gesungen und musiziert worden sei bis $10^{1} / 4$ Uhr.

$\mathrm{Um} 11 \mathrm{Uhr}$ ist ein Schuß von anderen Hausbewohnern gehört worden. $11 \mathrm{Uhr}$ 25 kehrte Herr $Z$. aus dem Theater zurück. Als er den Flur seiner Wohnung betrat, kam ihm Frau $N$. aus dem Schlafzimmer entgegen mit der Erklärung, ihr Kind Erika sei nicht wohl, sie habe soeben erbrochen. Sie forderte Herrn Z. auf, nach dem Ofen zu sehen; wobei Herr Z. auffiel, daß die Sprechweise der N. überstürzt, andererseits leicht gehemmt erschien.

11 Uhr $30 \mathrm{kam}$ das Dienstmädchen nach Hause. Frau N. öffnete dessen Schlafkammertür und sagte: „Schwärmer.“ Dies Wort klang dem Dienstmädchen so, als wenn Frau $\mathrm{N}$. angetrunken wäre.

Ein zweiter Schuß ist dann um $12^{3 / 4}$ Uhr gehört worden.

Im weiteren Verlaufe der Nacht, etwa gegen 4-5 Uhr hat man nochmals ein starkes Geräusch in dem Schlafzimmer der Frau N. gehört.

Am 7. Januar früh wurde in der Wohnung telephonisch angerufen und eine männliche Stimme wünschte Frau N. zu sprechen; darauf begab sich das Dienstmädchen zum Schlafzimmer der Frau N., und als sie auf wiederholtes Anklopfen keine Antwort erhielt, öffnete sie die nichtverschlossene Tür, fuhr aber sofort zurück, als sie beim Schein einer elektrischen Lampe eine männliche Person auf dem Rande eines der Betten sitzen sah. Sie benachrichtigte sofort Herrn Z., der im Zimmer folgende Situation vorfand: Der Fenstervorhang war zugezogen, vor dem Fenster brannte eine elektrische Tischlampe, die nach der Straße hin durch ein überhängtes Tuch abgeblendet war. Auf dem Bettrande des links am Fenster befindlichen Bettes befand sich in sitzender Stellung die Leiche seines Sohnes. Die Füße standen auf dem Bettvorleger; rechts von den Füßen befand sich eine größere Blutlache.

Der Rumpf war etwas vorn übergebengt. Der rechte Ellenbogen ruhte auf dem rechten Oberschenkel. In der rechten Hand lag ein Browning. Die Mündung der Waffe war gegen den geschlossenen Mund gerichtet, der sich etwa $25 \mathrm{~cm}$ über der Mündung der Waffe befand. Aus der Nase hing blutiger Schleim herab.

In der linken Hand befand sich eine $1 \frac{1}{2}$ Pfund schwere geleerte Kognakflasche, der Hals der Flasche war nach oben, der Boden der Flasche nach unten gekehrt, nach Angabe einzeiner Zengen soll sich der linke Arm im Ellbogen gewinkelt frei schwebend in der Luft befunden haben, nach anderen Angaben ist es möglich, daß der linke Ellenbogen dem linken Oberschenkel gestützt auflag. Die Gegenstände wurden in den Händen so fest gehalten, daß ihre Entfernung aus den Händen eine gewisse Kraft erforderte; insbesondere lag der Browning in der rechten Hand so fest, daß die Fingerbeere des rechten Zeigefingers auf dem Abzung der Waffe breit gedrückt erschien. 
Aus der Waffe waren 2 Schüsse abgefeuert worden; durch Selbsthemmung war die Waffe gesichert.

Links in dem Bett am Fenster lag die 10 jährige L. erschossen. Der Schul3 war vom linken Scheitelbein zur rechten Schläfe gegangen; dann war das Projektil in das Kopfkissen gedrungen. Ein Ausschuß war in dem Kopfkissen nicht vorhanden; eine größere Blutlache befand sich unter dem Kopfe. Rechts neben dem Fenster lag in dem anderen Bette erschossen die E. N. mit einem Schuß von der linken zur rechten Schläfe. Das Projektil wurde in einer wollenen Decke gefunden; auch im rechten Bett befand sich auf dem Kopfkissen eine große Blutlache.

Die Patronenhülsen lagen unter den Betten.

Frau N. wurde lebend, auf dem Fußboden, der Rumpf gegen einen Schrank gelehnt, gefunden.

Ein Nachtschränkchen war umgestürzt. Auf dem Teppich zu den Füßen des Schülers lag ein Handspiegel, so gelagert, daß der auf dem Bettrande sitzende Schüler vermutlich die Bewegungen der Hand bezw. der Waffe im Spiegel kontrollieren konnte, ferner eine Tasse, in der sich Reste eines weißen Pulvers befanden.

Flecke, von diesem weißen Pulver herrührend, befanden sich an dem Anzug des Schülers in Form länglicher Tropfspuren unterhalb der Kniee auf der Hose, reichlich weißes Pulver auf den Schuhen und auf dem Teppich.

Als Frau N. von dem eintretenden Herrn Z. gefragt wurde: Frau N. was haben sie gemacht? gab sie kaum Antwort. Sie griff mit der Hand wiederholt nach dem Kopfe und machte den Eindruek einer unbesinnlichen Person.

Ein herbeigerufener Arzt stellte starke Verengerung der Pupillen fest und schloß daraus auf Morphiumvergiftung.

Als die Transportkolonne erschien, un die Fran N. dem Krankenhause zuzufühen, antwortete sie auf die Frage nach der Bezahlung mit,, $\mathrm{j}^{26}$ und zeigte dabei auf sich.

Beim Heruntertragen sagte sie zu cinem der Transporteure auf der Treppe: „Lassen Sie mich nur nicht fallen".

Im Krankenhause angekommen, gab sie dem aufnehmenden Magistratsbeamten präzise kurze Antworten. Die aufnehmende Arztin bekundet, daß die N. bei der Einlieferung bei klarem Verstande war.

Nach einer Magenausspülung, bei der reichlich Potwein aus dem Magen der $\mathrm{N}$. entleert wurde, trat ein Zustand von BewuBtlosigkeit ein, das Gesicht wurde bläulich, der Puls klein, kaum zu fühlen, die Atmung aussetzend, die Pupillen blieben eng. Unter der Anwendung künstlicher Atmung und von O-Einblasungen besserte sich der Zustand innerhalb einer Stunde soweit, daß die Lebensgefahr beseitigt war.

Frau N. war dann bei klarem Bewußtsein und konnte geordnete Auskunft geben.

Die weiteren Ermittelungen ergaben das folgende:

An den Leichen der beiden Mädchen wurden - abgesehen von den Schuß3verletzungen - sonst keine weiteren Verletzungen vorgefunden. Die Mägen enthielten je 300-400 ecm Speisebrei; in beiden Mageninhalten wurde Morphium nachgewiesen.

Bei der Leiche des Schülers vermutete man eine Schußverletzung des Mundes; dafür schien die Haltung der rechten Hand mit der auf den Mund gerichteten Mündung der Waffe, die Lage des Spiegels auf dem Teppich am Fußboden, ferner dic Blutung aus der Nase zu sprechen.

Die Untersuchung der Leiche ergab aber keine Schußverletzung, auch sonst keine Zeichen von Verletzungen; vielmehr roch das Gehirn nach Eröffnung der 
Schädelkapsel stark nach Alkohol. Im Magen befanden sich nur ca. $30 \mathrm{~cm}$ Speisebrei, in dem Morphium nachgewiesen wurde. Speisebrei befand sich auch im Munde, zwischen den Zähnen, im Rachen, in der Speiseröhre und im Anfangsteile der Luftröhre, dessen Wände z. T. bekleidend; dagegen keine Speiseteile in den tieferen Teilen der Lunge. Es war also nichts aspiriert.

Das weiße Pulver in der Tasse, die weißen Flecke an der Hose, an den Stiefeln, auf dem Teppich bestanden aus Morphium. Hiernach mußte angenommen werden, daß der Tod infolge Alkohol- und Morphiumvergiftung eingetreten sei.

Das Morphium hatte Frau N. am 4. I. für 100 M. erworben. In Zimmer wurde ferner eine Rekordspritze mit abgebrochener Nadel vorgefunden, ca. $10 \mathrm{ccm}$ fassend. Das Innere der Spritze war mit einem weißen Belag von Morphiumpulver ausgekleidet. Auch diese Spritze hatte Frau N. kurz vor dem hier geschilderten Vorgang gekauft. Frau $N$. besa $ß$ ferner eine nicht unerhebliche Menge Arsenik, wovon jedoch im Mageninhalt des Schülers nichts gefunden wurde, und schließlich Veronal.

Vor dem Untersuchungsrichter erklärte Frau N.: Sie habe nicht nur mit dem Schüler, sondern auch mit einem Kaplan Geschlechtsverkehr gehabt, demselben, der sie am 7. I. morgens telephonisch angerufen habe. Der Schüler sei auf den Kaplan eifersüchtig gewesen. Er habe ihr deshalb wiederholt Szenen gemacht, so besonders am $\mathrm{Abend}$ des 6 . I. Sie selbst habe gefürchtet, das Liebesverhältnis mit dem Schüler werde dem Vater des letzteren, ihrem Wirt, bekannt werden; sie habe auch eine Anzeige an den Bischof gefürchtet.

Am Abend des 6. I. habe sie infolge der Aufregungen Kopfschmerzen gehabt und Aspirin nehmen wollen. Der junge $Z$. habe eine Flasche Burgunder geholt, er habe das Aspirin in den Rotwein getan und sie habe es aus einem Glase getrunken. Der Schüler habe aus der Tasse getrunken. Bald nach dem Genusse des Rotweins sei sie bewußtlos geworden; sie könne daher über das weitere Geschehen keine Auskunft erteilen; sie sei erst wieder zu sich gekommen, als die Transporteure.sie frühmorgens nach dem Krankenhause gebracht hätten.

Über das Vorleben der Frau N. ließ sich feststellen, daß sie sich seit Mai v. J. zusammen mit ihrem Manne in der gedachten Provinzialstadt aufgehalten hatte und zunächst bei einem Apotheker gewohnt hatte; durch den Sohn desselben hatte Frau N. den Schüler Z. kennen gelernt und ihn gesehlechtlich verführt. Auf Betreiben des Schülers Z. ist Frau N. mit ihren beiden Töchtern in die Wohnung des $Z$. gezogen.

Ihrem Mann hat Frau N. die eheliche Treue nicht gehalten, ebensowenig der Ehemann seiner Frau. Seit Anfang Januar war das Bankguthaben der Frau. N. erschöpft, sie war mittellos.

Vielleicht konnte es auch zweifelhaft erscheinen, ob der Ehemann aus Brasilien zurückkehren würde.

Es wurde noch festgestellt, daß Herbst 1910 der Schwiegervater, September 1911 die Schwiegermutter, September 1912 die Mutter, Februar 1914 der Vater gestorben waren.

Frau N. wird als eine kokette, höchst sinnliche, hysterische Person geschildert. Sie hatte früher lesbischer Liebe gehuldigt. Ein Zeuge berichtet, daß sie nach dem Beischlafe in Zuckungen verfiel, ein anderer Zeuge, daß sie während des Beischlafes an den Brüsten des Mannes zu saugen versuchte.

Schließlich sei noch bemerkt, daß sie dem Schüler $Z$. aus der Hand geweissagt hat, er würde aus Liebe zu einer verheirateten Frau sterben; bei anderer Gelegenheit hat sie geäußert, wenn sie aus dem Leben ginge, nehme sie ihre Kinder mit.

Nach der Geschichtserzählung darf angenommen werden, daß Frau N. mit ihren Kindern sterben wollte. Um den Verdacht der Täterschaft von sich abzu- 
lenken, hat sie den Schüler Z. mitvergiftet und ihm den Browning in die Hand gedrückt.

Von der Staatsanwaltschaft wurde nun unter anderem dem Gerichts. arzte die Frage vorgelegt, ob der Tod des Schülers $Z$. in der Stellung eingetreten sein könne, in der die Leiche gefunden wurde. Ich habe dazu etwa folgende Ausführungen gemacht:

I. Die Frage, ob der Tod des Z. in der Stellung eingetreten sei, in der die Leiche gefunden wurde, kann nur beantwortet werden nach Erledigung der Vorfrage, wann und in welcher Weise wohl der Tod des $Z$. eingetreten sein mag.

Da der Mund keinerlei Schußverletzungen zeigte, so ist der Eintritt des Todes durch einen SchuB in den Mund ausgeschlossen. Vielmehr ist als Todesursache Alkohol- und Morphiumvergiftung anzunehmen. Hierfür sprechen folgende Umstände und Erwägungen: das Gehirn roch nach Alkohol und im Magen wurde Morphium nachgewiesen.

Bis $10^{1} / 4$ Uhr ist Musik und Gesang gehört worden, dann wird Frau N. mit $Z$. in das Schlafzimmer gegangen sein.

Wo befand sich nun Z., als er das Gift nahm?

Ich nehme an, daß er das Gift auf dem Bettrande sitzend getrunken hat, bzw. dort in einen schlafsüchtigen Zustand (Narkose) verfallen ist. Wäre Z. an einer andern Stelle des Zimmers in den Zustand der Narkose geraten, so hätte Frau Z. den bewußtlosen N. zu Bett tragen und ihn dort hinlegen müssen.

Es ist aber bekanntlich für eine einzelne Person ganz außerordentlich schwer, den Körper eines erwachsenen, über einen Zentner schweren Menschen zu heben und zu tragen. Die Kräfte einer Frau dürften dazu kaum ausreichen.

Es liegt daher die Annahme nahe, daß Z., während er auf dem Bettrande saß, allmählich in die Narkose verfiel.

Nun steht weiter fest, daß Z. Erbrechen hatte.

Nicht Erika hatte erbrochen, wie Frau N. angibt; der Magen der beiden Kinder enthielt je $300 \mathrm{ccm}$ Speisebrei; es fand sich bei ihnen kein Erbrochenes in der Speiseröhre oder im Munde, wohl aber fand sich bei Z. das Erbrochene im Munde, zwischen den Zähnen, in der Speiseröhre und in dem oberen Teile der Luftröhre an den Wänden derselben.

Noch ein Weiteres von Wichtigkeit: Bei Z. war das Erbrechen ein terminales, wären nämlich nach dem Brechakt noch einzelne Atemzüge erfolgt, so hätte das Erbrochene bis in die feineren Luftwege eingeatmet werden müssen, davon ist aber nichts festgestellt. Der Tod ist während des Erbrechens eingetreten.

Frau N. hat das Erbrochene in einem Handtuche aufgefangen und das Handtuch in die schmutzige Wäsche gelegt. 
Falls Z. nur einmal erbrochen hat, würde anzunehmen sein, daß er schon 11 Uhr 25 Minuten, als der Vater nach Hause zurückkehrte, tot gewesen sei, denn bei der Heimkehr des Herrn Z. erzählte Frau N., daß ihre Tochter Erika gebrochen habe.

Nimmt man dagegen an, daß $Z$. mehrmals erbrochen habe, so würde zu folgern sein, $d a B$ Frau N. sich wahrscheinlich erst nach dem Tode des Z. dem dritten Opfer zugewandt haben wird. Da der zweite Schuß $12^{3} / 4$ Uhr fiel, würde der Tod des Z. spätestens auf $12^{3} / 4$ anzunehmen sein.

Die Zeit des Todes des Z. fällt demnach zwischen ca. $11 \mathrm{Uhr} 10 \mathrm{Mi}$ nuten und zwischen $123 / 4 \mathrm{Uhr}$.

Daraus geht hervor, dal bis morgens $1 / 28 \mathrm{Uhr}$, als die Leiche gefunden wurde, 6-7 Stunden verstrichen sein werden. In dieser Zeit kann die Totenstarre sich vollständig entwickelt haben, wie man das auch sonst bei Leichen sieht, die allmählich im Laufe von $4-6$ Stunden erstarren.

Wie groß freilich die Dosis Alkohol und wie groß die Dosis Morphium gewesen ist, die $Z$. erhalten hat, ist nicht mehr festzustellen.

Daß die Menge Alkohol sehr groß gewesen sein muß, geht daraus hervor, daß das Gehirn nach Alkohol roch, daß die Morphiummenge ebenfalls sehr groß gewesen sein muß, lehrt ein Blick auf die am Boden liegende Tasse, die im Innern, in halber Höhe, einen durch Rotwein geröteten Rand und einen Bodensatz von ca. einem gestrichenen Teelöffel voll Morphium enthielt.

Man gewinnt dadurch, so wie durch die zahlreichen Flecke auf den Stiefeln, so wie auf der Hose (die evtl. noch auf Morphium zu untersuchen wären), den Eindruck, daß das Morphium aufs Geratewohl in den Wein hineingeschüttet und getrunken wurde.

Es wäre von Interesse gewesen, zu wissen, wieviel Morphium das Erbrochene im Munde und das Erbrochene in dem Handtuche enthielt, und wieviel Morphium in der Leiche vorhanden war.

Quantitative Bestimmungen liegen nicht vor.

Nimmt man an, daß der Tod des Z. bis 11 Uhr 10 Minuten eingetreten ist, so würde die Vergiftung innerhalb einer knappen Stunde zum Tode geführt haben; trat dagegen der Tod 12 Uhr 45 Minuten ein, so wäre bis zum Eintritt des Todes ca. $2^{1 / 2}$ Stunde vergangen.

Beide Zeiträume sind als ausreichend anzusehen, sowohl für clen Verlauf einer akutesten Alkohol- wie Morphiumvergiftung. In der gerichtsärztlichen Literatur sind Fälle bekannt, in denen die Morphiumvergiftung innerhalb $1^{1 / 2}$ Stunde, einmal innerhalb 2 Stunden eintrat (Maschka, Bd. 2, S. 422). Man wird aber im vorliegenden Falle nicht nur mit einer sehr großen Dosis Morphium, die wahrscheinlich im berauschten Zustande per os verabfolgt wurde, zu rechnen haben; es besteht vielmehr die Möglichkeit, daß gleichzeitig eine oder mehrere 
Morphiumeinspritzungen gemacht wurden. Unter den Asservaten befindet sich eine Rekordspritze mit abgebrochener Nadel, die im Innern einen milchweißen Beschlag von Morphiumpulver zeigt. Freilich wurde bei $Z$. während der Obduktion keine Einstichstelle gefunden. Es muß indessen hervorgehoben werden, daß eine solche Stichstelle dem Auge des Arztes entgehen kann. Würde aber eine Morphiumeinspritzung gemacht worden sein, so würde es verständlich sein, daß der Tod noch schneller eintrat, als bei alleiniger Vergiftung per os.

Bei schwerer Alkoholvergiftung kann der Tod schon nach $1 / 2$ bis 1 Stunde eintreten.

Genossen wurde $1 / 3$ Flasche Kognak und 1 Flasche Burgunder, wobei allerdings nicht feststeht, wieviel etwa von dem Wein auf den Teppich verschüttet worden sein mag. Von dem Burgunder hat auch Frau N. getrunken, denn es wurde am andern Morgen reichlich Rotwein bei der Magenausspülung zutage gefördert.

Man rechnet, daß ca. 300-500 g unserer Branntweinsorten den Tod eines erwachsenen Menschen veranlassen können. Sollte Z. den gesamten Kognak ( $1 / 3$ Flasche) getrunken haben, dann hätte er jedenfalls eine stark toxische Dosis erhalten.

Einer besonderen Erörterung bedarf nun die festgestellte Körperhaltung der Leiche.

Die Leiche wurde auf dem Bettrande sitzend gefunden. Der linke Arm schwebte gewinkelt im Ellenbogengelenk frei in der Luft, nach anderer Angabe war er auf den linken Oberschenkel gestützt. Die linke Hand hielt eine leere Kognakflasche von 11/2 Pfund Gewicht; der rechte Arm war auf den rechten Oberschenkel gestützt. Die rechte Hand hielt eine Browningpistole, der Lauf war nach dem Munde des Z. gerichtet. Im Laufe des Vormittags ist dann der Rumpf der Leiche vornübergesunken, auch hat sich die Stellung der rechten Hand verändert, so daß die Photographie nicht mehr das ursprüngliche Bild naturgetreu wiedergibt.

Ich nehme an, daß Frau N. den Erbrechenden aufgesetzt hat und zwar in der Weise, daß sie die Ellenbogen auf seine Oberschenkel stützte.

Die vornübergebeugte Haltung des Sterbenden kann dadurch wahrscheinlich gemacht werden, daß sich auf dem Teppich Flecke befanden, die von dem Erbrochenen herrühren können; ferner spricht für diese Haltung der Umstand, daß sich an den Stiefeln zahlreiche Blutspritzer befanden. Es ist danach anzunehmen, daß das Blut aus der Nase auf den Teppich herabgetropft ist.

In clieser sitzenden Stellung muß der Tod eingetreten sein.

Eine derartige Körperhaltung, die anscheinend die letzte vitale Bewegung festhält, bezeichnet man mit dem Namen der kataleptischen Totenstarre. Die Bezeichnung ist sehr unglücklich, denn weder hat 
dieser Zustand etwas mit Katalepsie zu tun, noch mit der Totenstarre. Man muß vielmehr für solche Fülle annehmen, daß die letzte vitale Muskelkontraktion über den Tod heraus festgehalten wurde und daß dann erst allmählich, nach einer gewissen Zeit, die Totenstarre eintrat. Die Franzosen unterscheiden den Spasme cadavérique von der Rigidité cadavérique.

Diese beiden Vorgänge sind scharf auseinanderzuhalten. Gebraucht man die Bezeichnung kataleptische Totenstarré, so läuft man Gefahr, den Vorgang mit der Leichenstarre, wie sie im Laufe einiger Stunden bei jeder Leiche einzutreten pflegt, zu konfundieren.

Beim Durchmustern der sehr umfangreichen Literatur gewinnt man die Überzeugung, daß die Totenkatalepsie (Leichenspasmus) etwas ganz anderes ist, als die Totenstarre, die in der regulären Nystenschen Reihenfolge auftritt.

Es müssen hier Verhältnisse vorliegen, die im ganzen Körper oder in einzelnen Teilen desselben zu einer plötzlichen Erstarrung der quergestreiften Muskulatur führen. Welches die Bedingungen sind, unter denen dies geschieht, kann uns die Physiologie vorläufig nicht sagen. Wir wissen nur, daß die Totenkatalepsie im Anschluß an Schußverletzungen des Gehirns eintreten kann. Eine solche lag hier nicht vor. Es ist ferner bekannt, daß sie im Anschlu $B$ an eine Erstickung eintreten kann. Eine plötzliche Erstickung nehme ich für den von mir untersuchten Fall nicht an; denn es lag kein Bolustod vor. Hätte $Z$. nur noch einige kräftige Atembewegungen ausgeführt, so würde er den Speisebrei entweder ausgehustet oder aspiriert haben. Ein plötzlicher Tod könnte in unserem Falle möglicherweise eingetreten sein und den Ablauf der Vergiftungssymptome unterbrochen haben. Anatomische Abweichungen, die für einen plötzlichen Tod sprechen würden, haben sich aber nicht ergeben. Ich halte einen plötzlichen Tod (Herztod) auch nicht für wahrscheinlich.

Wir wissen aber, daß die kataleptische Totenstarre sich an Krampfzustände des Körpers (Tetanus, Tetanie) anschließen kann.

Es wäre denkbar, daß im vorliegenden Falle lokale oder allgemeine Krämpfe eingetreten wären, und daß die vornübergebeugte Haltung, die Ellenbogen auf die Knie gestützt, durch die schnell eintretende Totenstarre fixiert worden wäre.

Daß Krämpfe bei der Alkoholvergiftung auftreten können, ist außer Zweifel; bezüglich der Opium- und Morphiumvergiftung sagt $\mathrm{H}$ usemann (vgl. Maschka, Handbuch II; 1882, S. 423), daß Krämpfe in verschiedenen Muskeln vorkommen, namentlich bei Kindern, mitunter in höchst ausgeprägter Weise bei Erwachsenen.

In dem Falle von Pella c a ni (1885) trat bei einem 21 jährigen Manne nach Injektion von 0,02 Morphium und 0,002 Atropin Steifigkeit in 
den Masseteren auf, Trismus, Steifigkeit der Nackenmuskeln, Unmöglichkeit, den Hals zu bewegen, dann allgemeine tonische Konvulsionen mit vorwiegendem Opisthotonus.

Krampfzustände können gelegentlich also bei einer Morphium- bzw. Alkoholvergiftung eintreten. Es ist aber meines Erachtens gänzlich ausgeschlossen, daß während des Ablaufes einer Alkohol- und Morphiumvergiftung, das heißt während der Narkose, eine Flasche und ein Revolver dauernd in den Händen festgehalten werden können. Die Muskeln erschlaffen während der Narkose vollständig, den Händen müßten diese Gegenstände entgleiten; dabei gehe ich von der Voraussetzung aus, daß das Morphium per os oder durch subcutane oder intramuskuläre Injektion verabfolgt wurde. Der ungünstigste Fall würde der sein, daß die Spitze der Injektionsnadel zufällig in eine Vene gelangt und daß das Morphium direkt in den Kreislauf eingespritzt wäre. Fïlle, in denen medizinale Dosen von Morphium intravenös injiziert wurden, sind von $\mathrm{Nussbaum}$ und Feith mitgeteilt worden.

Am wertvollsten sind die Beobachtungen von $\mathrm{N}$ uss ba u m, der, wie er mitteilt, an sich selbst ca. 2000 subcutane Morphiuminjektionen vorgenommen hat. Dreimal kam es vor, daß die Nadelspitze in eine Vene geriet.

Ein starkes Stechen und Brennen überlief wie ein Blitz die ganze Haut vom Scheitel bis zur Sohle; das Gesicht wurde dunkelrot, Ohrensausen, Funkensehen, essigsaurer Geschmack auf der Zunge (Morph. acet.) traten auf. Der Puls war enorm beschleunigt auf 160-180 Schläge pro Minute. N. hatte das Gefühl, als ob von den jagenden Pulsen die Brustwand durchrannt, das Trommelfell durchstoßen, der Augapfel aus seiner Höhle bei jedem Pulse herausgeschleudert würde. Dieser bedrohliche Zustand dauerte 8 Minuten, Totenblässe des Gesichtes folgte für 1 Stunde.

Nach 2 Stunden war nicht der geringste Nachteil vorhanden.

Bei 3 Kranken Nussbaums, bei denen ebenfalls zufällig die Morphiuminjektion zu einer intravenösen wurde, verlief der Vorgang noch bedenklicher. Es mangelte teilweise das BewuBtsein und es traten Konvulsionen ein.

Über die klinischen Erscheinungen bei intravenöser Injektion letaler Dosen liegen beim Menschen keine Beobachtungen vor. Tierversuche wird man zur Klärung kaum heranziehen dürfen, da Tiere anders auf Morphium reagieren als der Mensch.

Für sehr wahrscheinlich möchte ich eine zufällige intravenöse Injektion des Morphiums in unserem Falle nicht halten und zwar deshalb nicht, weil derartige zufällige intravenöse Injektionen ungemein selten sind. Bei den vielen Hunderten und Tausenden von Morphiuminjektionen, die täglich gemacht werden, ist doch seit $\mathrm{Nussbaum}$ nichts wieder von üblen Zufällen durch zufälliges Hineingelangen der Punktionsnadel in eine Vene bekannt geworden. Dazu kommt, da.B im vorliegenden 
Falle die Nadel abgebrochen gefunden wurde; das erweckt den Eindruck, daß die Einspritzung beim ersten oder bei dem wiederholten Versuche nicht gelang, möglicherweise deshalb nicht, weil sich reichlich ungelöstes Morphium in der Spritze befand und dieses die Kanüle verstopfen konnte.

Aber selbst wenn wir annehmen, daß eine letale Dosis Morphium intravenös injiziert wäre, so würden doch die klinischen Erscheinungen zunächst wie in den nicht-letalen Fällen mit blitzartig über den Körper laufendem Brennen und Stechen, Klopfen der Gefäße, jagendem Puls, Ohnmacht beginnen.

Auch in diesem Zustande würde $Z$. nicht andauernd einen Revolver in der rechten Hand und eine Kognakflasche in der linken Hand gehalten haben können. - Die Frage der kataleptischen Totenstarre hat schon einmal in einem Kriminalfalle eine wesentliche Rolle gespielt. Es ist das der von Marc berichtete Fall Courbon (Annales d'hygiène publique 7 ; 1832).

Die Leiche des Courbon, cines Trinkers, wurdo in einer Grube von 4-5 FuB Lünge und Breite und einer Tiefo von $2^{1 / 2}$ Fuß gefunden (S. 616), in solcher Haltung, daß der Kopf stark auf die Brust gencigt, den tiefsten Punkt in der Grube bildete. Der Ruicken und das Becken schwobten frei in der Luft, und nur die Spitze des linken Fußes, des rechten Knies und die Spitze des rechten Fußes berührten die Wände der Grube.

Auf Grund eines angeblich belauschten Gespräches waren 2 Personen, als des Mordes verdächtig, in Haft genommen und zu lebenslänglicher Zwangsarbeit verurteilt worden.

In dem von Richond und Fodéré aus Straßsburg eingeholten Gutachten wird auseinandergesetzt, daß kein Grund vorliegt, an ein Verbrechen zu denken, daß es sich vielmehr mit grölter Wahrscheinlichlseit um einen plötzlichen Tod aus inneren Ursachen (bei cinem Alkoholiker) gehandelt hat und daB in solehen Fällen manchmal - eine raideur spasmodique - ein plötzlicher Spasmus der Muskeln vorkommt, der zu bizarren Haltungen des Körpers Anlaß geben kann. Die obduzierenden Ärzte hatten an die Möglichkeit einer Halswirbelluxation, Zertrümmerung des Rïckenmarks und tetanische Kontraktion des Muskeln gedacht.

Nach 2 Jahren wurde das Urteil kassiert und die Verurteilten wurden in Freiheit gesetzt.

Auf diesen letzten Fall bin ich durch Herrn cand. med. Baumann aufmerksam gemacht worden, dem ich dafür meinen Danlk aussprechen möchte. Er bildet mit dem von Wahncau beobachteten Falle, in dem eine an Schrumpfniere leidende Frau tot in der Ecke eines Hofes stchend aufgefunden wurde, die einzige Beobachtung von liataleptischer Starre bei chronischen Alkoholisten. Akute Alkoholvergiftung mag öfter, besonders in Fällen von Selbstmord, vorgelegen haben, die Literatur enthält darüber keine Angaben.

Von kataleptischer Starre bei Morphiumvergiftung ist überhaupt nichts bekannt.

Ich nehme also in dem von mir beobachteten Falle an, daß die Täterin den Sterbenden beim Brcchakt aufgesetzt hat und daß dann 
eine kataleptische Starre bzw. eine schnell eintretende, frühe Totenstarre die sitzende Haltung fixiert haben kann; erleichtert wird die Fixierung der Stellung dadurch sein, daß die Ellenbogen den Oberschenkeln auflagen.

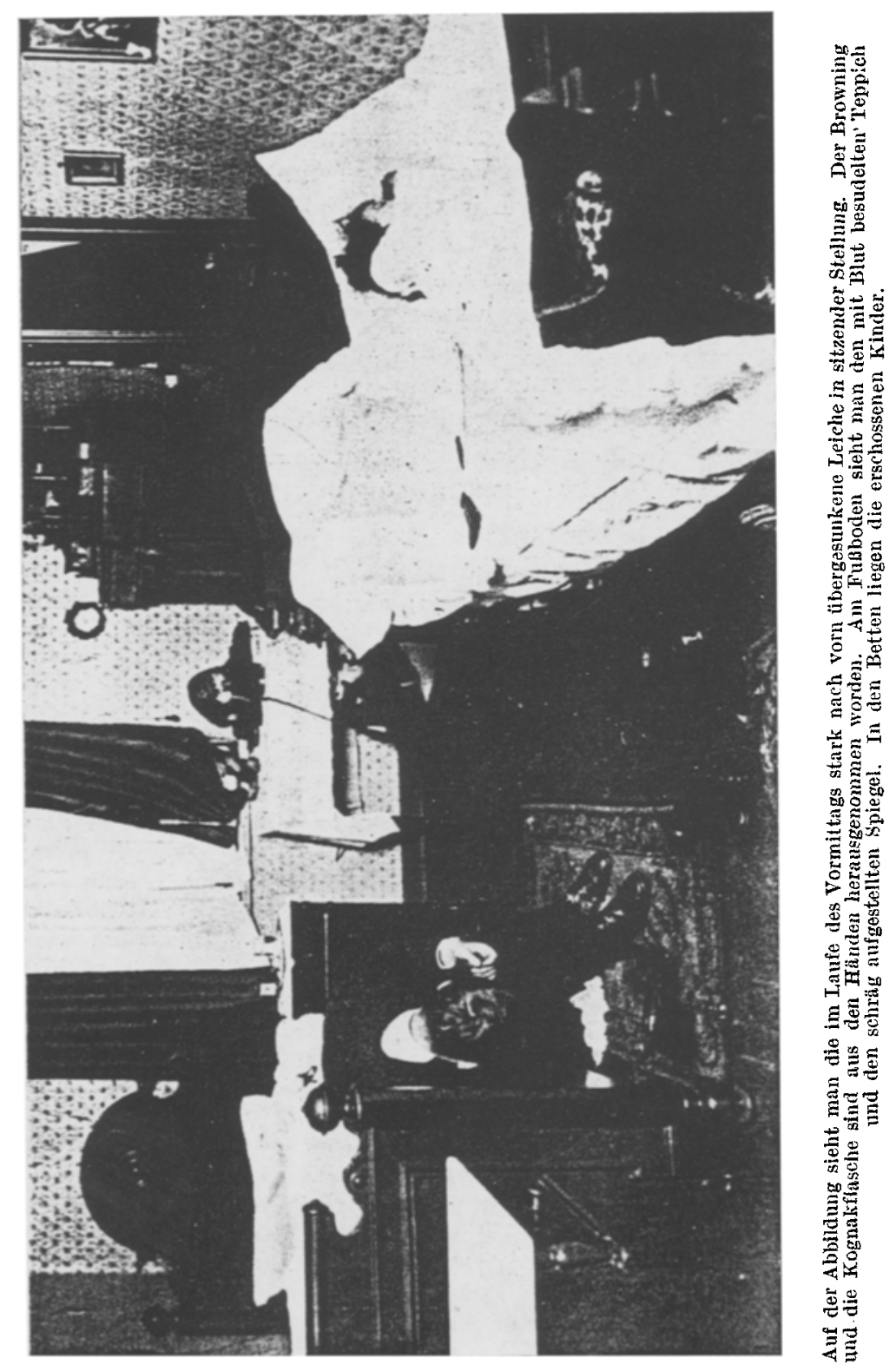


$11 \pm$ Th. Lochte: Ein Fall von absichtlich vorgetäuschter kataleptischer usw.

Eine langsam eintretende Totenstarre möchte ich deshalb ausschließen, weil dann die Täterin den Körper eine oder mehrere Stunden lang gestützt haben müßte. Das erscheint recht wenig wahrscheinlich.

Daß bis zum Eintritt des Todes die Kognakflasche und der Browning dauernd in der Hand gehalten wurde, nehme ich nicht an und zwar deshalb nicht:

1. weil bei länger anhaltender Morphium- und Alkoholnarkose die Muskulatur erschlafft und das Festhalten in der Hand unmöglich ist;

2. weil bei schnellerem Tode (nach intravenöser Morphiuminjektion) im Hinblick auf die Reihenfolge der Vergiftungssymptome Z. die Gegenstände wahrscheinlich nicht nach der Injektion in der Hand gehalten haben wird;

3. weil der ganzen Situation die innere Wahrscheinlichkeit fehlt. $Z$. hatte überhaupt keinen Grund, sich zu vergiften oder zu erschießen. Zeichen von Lebensüberdruß hat er niemals geboten; den Kindern war er sehr zugetan. Es ist meines Erachtens ausgeschlossen, daß er diesen etwas getan haben sollte.

Wenn Frau N. den Selbstmord des Z. vortäuschen wollte, so mußte sie das natürlich möglichst sinnfällig machen. Sie legte den Spiegel auf den Fußboden, drückte den Browning in die rechte $\mathrm{Hand}$; schließlich die Kognakflasche in die linke Hand, um als Grund des Selbstmorcles auf die Berauschung aufmerksam zu machen.

Sie übersah dabei aber, daß jemand, der im Begriffe ist, sich zu erschießen, bestimmt die Kognakflasche vorher aus der Hand legen. wird.

\section{Literatur.}

Brown-Séquard, Recherches expérimentales montrant que des causes diverses, mais surtout des lésions de l'encéphale, et en particulier du cervelet, peuvent déterminer, après la mort, une contracture générale ou locale. Comptes rendus de l'Académie des Sciences 93, II, 1149. 1881. - Brown-S èquard, Attjtudes après la mort. La Nature 1884, S. 405. - Jules Ronyer, Attitudes après la mort. La Nature 1884, S. 57, II. - Fernand Mazellier, Du spasme cadavérique. These de Lyon 1897. - Marc, Du procès en condemnation, révision et réhabilitation de Regis Rispal ... et Jacques Gallond. Annales d'hygiène publique 1832, T. VII, S. 569-621. - Etienne Martin, De la rigidité cadavérique, dans les membres atteints de contracture pendant la vie. Archives d'anthropologie crim 13, 536. 1898. - J.J. Matignon, Trois observations de ,catalepsie post mortem". trchives d'anthropologie criminelle $2 t, 444$. 1912. - John Brinton, On instantaneous Rigor, as the occasional Accompainment of Sudden and violent Death. Amer. Journ. of Med. Sciences 1870, S. 87. - T. Long more, On the Perpetuation of attitude and facial Expression which is occasionally met with in Soldiers who have been killed by gunshot on fields of battle. Army medical Departement Report for 1870. Vol. XII. London 1872, S. 283. - Nussbau m, Die Gefahren der subcutanen Injektion. Ärztl. Intelligenzblatt, München 3, 9. 1865. - A. Mühe, Ärztl. Intelligenzblatt, München 1865, S.690. - Hand- und Lehrbücher der gerichtl. Medizin. 